\title{
Modulation bandwidth and noise limit of photoconductive gates
}

\author{
J. R. HWANG, H. J. CHENG*, J. F. WHITAKER \\ Center for Ultrafast Optical Science, University of Michigan, Ann Arbor, \\ MI 48109-2099, USA
}

J. V. RUDD

Picometrix Inc., P.O. Box 130243, Ann Arbor, MI 48113, USA

Received 8 November 1995; revised 18 January; accepted 26 January 1996

The modulation bandwidth and noise limit of a photoconductive sampling gate are studied by reducing the parasitic capacitance and leakage current of the sampling circuit using an integrated junction field-effect transistor (JFET) source follower. The modulation bandwidth of the photoconductive sampling gate is limited by the external parasitic capacitance, and its efficiency is found to saturate at a laser gating power of about $1 \mathrm{~mW}$. It is determined that the noise of the photoconductive sampling gate is dominated by the photovoltaic current due to the gating laser amplitude fluctuation. A minimum noise level of $4 \mathrm{nV} \mathrm{Hz}^{-1 / 2}$ has been measured, and an enhancement in signalto-noise ratio by a factor of $>45$ has been achieved after the integration of the source follower with the photoconductive sampling gate. The JFET source follower serves to increase the modulation bandwidth of the photoconductive sampling gate by about 15 times and buffer the charge of the measured signal using its extremely high gate input impedance. The performance of the photoconductive sampling gate in regard to invasiveness and gating efficiency has been optimized, while a picosecond temporal resolution has been maintained and the signal-to-noise performance has been enhanced using a gating laser power as low as $10 \mu \mathrm{W}$.

\section{Introduction}

Progress in ultrafast photoconductive (PC) detectors based on short-carrier-lifetime semiconductors has led to the development of devices that have subpicosecond response when illuminated by ultrafast, pulsed lasers. Due to the extremely large bandwidth of these high-speed optoelectronic devices, there are no conventional electronic techniques with the capability to measure their response. However, optically-based sampling techniques, such as electrooptic (EO) sampling [1], do have a faster response than these short-carrier-lifetime PC materials, and thus have sufficient measurement bandwidth to characterize these devices. A second technique for characterization of these devices is photoconductive sampling, where a second PC switch is used to gate and measure short-duration electrical signals $[2,3]$.

Although the PC sampling gate does not have as high a measurement bandwidth as the

*Current affiliation: Picometrix Inc., P.O. Box 130243, Ann Arbor, MI 48113, USA. 
transducer in an EO measurement, this sampling technique has a much higher sensitivity than the EO method. For instance, an interdigitated, metal-semiconductor-metal (MSM) detector [4] has been monolithically integrated with a similar PC sampling gate on an ultrafast response, low-temperature-grown-GaAs substrate (LT-GaAs) [5] to form an optical-waveform analyser. This gate has been demonstrated by Chen et al. [6] to have a $1.2 \mathrm{ps}$ resolution and $500 \mathrm{pW}$ noise equivalent power (NEP). Photoconductive sampling gates have also been fabricated on freestanding probes $[7,8]$ which are being developed for integrated circuit testing [9]. When combined with a scanning force microscope (SFM) [10] or scanning tunnelling microscope (STM) [11], PC probes offer submicrometre spatial resolution and picosecond temporal resolution for device or circuit measurements.

The temporal resolution of a PC sampling measurement is generally determined from the convolution of the responses of two switches. However, it is essential to this investigation to realize that this is not simply an autocorrelation experiment. Although a switch and a gate may be integrated on the same substrate with the same electrode pattern, photoexcited charges in the different elements have different transport properties depending on the bias conditions and photogenerated carrier densities. For instance, a detector is often biased by a direct current (d.c.) high-voltage source which has a low impedance and can supply a large current. Since the bias field in a typical detector is high, the photoexcited carriers are driven to move at their saturation velocity soon after generation. The amplitude of the detector response is essentially linearly proportional to the exciting laser power. On the other hand, a PC sampling gate is biased by a transient electrical signal, which is typically a small output voltage from a detector with a high output impedance.

One problem with PC sampling is that in order to increase signal-to-noise ratio, a milliwatt or more of laser power is often used to increase the conductance of the gate. However, the gating efficiency may not be linearly proportional to the gating laser power, since the transient electrical signal from the detector can supply only a limited current as mentioned above. Also, the screening effect of the PC space charge is not negligible when a high photoexcited carrier density exists [12]. For free-standing sampling probes which may be used in active-circuit measurements, other side effects must be taken into consideration. For example, driving a PC sampling gate to an extremely low impedance may lead to a situation where the probe is too invasive for a device-under-test, extracting an excessive amount of charge. The heating of the PC sampling gate and device-under-test and the scattering of the high gating laser power may also need to be considered.

From the standpoint of noise, the major noise source in a detector is the dark current at room temperature. For the PC sampling gate, however, photovoltaic current generated at the imperfect ohmic contacts by the gating laser amplitude fluctuation appears to be the dominant noise source [13]. In general, the laser-induced noise has a $1 / f$ spectrum at low frequency and can be reduced by increasing modulation frequency $[14,15]$. However, the signal amplitude also drops at high modulation frequency. The overall signal-to-noise ratio is typically optimized at a modulation frequency below $1 \mathrm{kHz}$.

In this paper, the parasitic capacitance of the sampling system, including the PC sampling gate, the coaxial cable and the data acquisition electronics, is found to reduce the signal-modulation frequency product of the circuit and degrade the signal-to-noise ratio of the device. By using a novel JFET-based source follower configured with the PC sampling gate, the modulation bandwidth of the optoelectronic sampling circuit has been increased by about 15 times and the signal-to-noise ratio has been enhanced by about 45 times. The noise limits of the PC sampling gate have also been characterized down to a level of $4 \mathrm{nV} \mathrm{Hz}^{-1 / 2}$. The gate noise is found 
to be linearly proportional to the square root of the gating laser power and has a $1 / f$ frequency spectrum, while the gating efficiency does not increase linearly with the gating laser power for values over $10 \mu \mathrm{W}$. Absolute-voltage measurement capability and the invasiveness of the PC sampling gate are also discussed.

\section{Experimental setup}

A schematic diagram of the experimental configuration used to determine the modulation bandwidth and noise of a PC sampling circuit is shown in Fig. 1. To distinguish the PC sampling gate from the gates of the JFET devices, the former will be referred to as the photogate. Both the detector and the photogate utilized are PC switches with similar interdigitated MSM structures. The detector is a Picometrix PX-D14 device with a full-width half-maximum (FWHM) temporal response of $\sim 9 \mathrm{ps,} \mathrm{while} \mathrm{the} \mathrm{photogate} \mathrm{has} \mathrm{a} 4$ ps temporal response, a $4 \mathrm{pF}$ capacitance and a $0.008 \mathrm{~A} \mathrm{~W}^{-1}$ d.c. responsivity at $5 \mathrm{~V}$ bias [16]. They are each packaged in separate modules with coaxial connectors for bias and input and output of electrical signals. When connected, short-duration waveforms from the detector travel about $1 \mathrm{~cm}$ to the photogate. A mode-locked $\mathrm{Ti}$ : sapphire laser operating at $810 \mathrm{~nm}$ wavelength with a repetition rate of $82 \mathrm{MHz}$ produces $100 \mathrm{fs}$ duration pulses to drive both $\mathrm{PC}$ elements.

The laser output is split into two beams, with one delayed with respect to the other by use of a moving mirror. The first beam, modulated by an acoustic-optic (AO) modulator at frequencies between $1 \mathrm{~Hz}$ and $80 \mathrm{kHz}$, excites the switch in the detector module and generates $\sim 9$ ps electrical pulses used as the signals to be measured. The second beam consists of the pulses which activate the photogate, so that the electrical signals may be sampled as they propagate past this element. The gating laser power is varied from $5 \mathrm{~mW}$ down to $0.5 \mathrm{nW}$ ( $60 \mathrm{pJ}$ per pulse to $6 \mathrm{aJ}$ per pulse). A packet of charge, proportional to the convolution of the electrical pulse to be measured and the impulse response of the photogate at a given time delay between the two laser pulses, is injected into the gate of the source follower at the repetition rate of the laser. The output voltage of the source follower can be measured using a lock-in amplifier or a digital averaging oscilloscope. For comparison, the signal directly from the photogate module without the source follower was also measured.

The source follower is assembled using a pair of matched, $n$-depletion JFETs (model

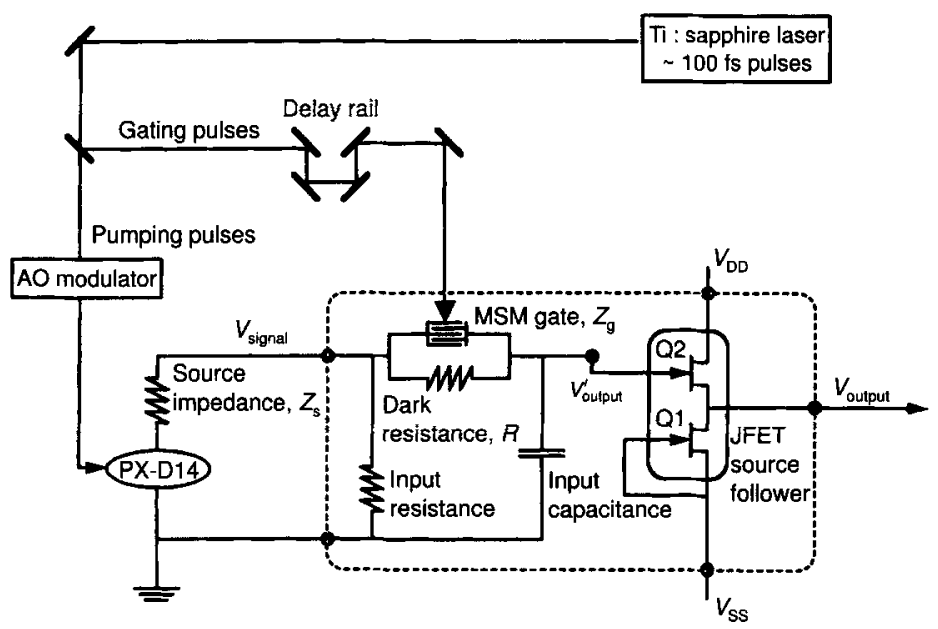

Figure 1 Schematic diagram of the photoconductive gating experiment. 
2N5912). The gate-source pinch-off voltage of this transistor is about $2 \mathrm{~V}$. The first transistor, Q1 (Fig. 1), serves as a constant current source with the source and gate pins tied together, while the second transistor, Q2, acts as the source follower. The bias voltages, $V_{\mathrm{DD}}$ and $V_{\mathrm{SS}}$, are supplied by two $9 \mathrm{~V}$ batteries. As a matched pair of transistors, the source voltage of JFET Q2 follows its gate voltage [17]. Both JFETs are operated in their saturation region. The short-circuit, common-source input capacitance, $C_{\mathrm{iss}}$, of one JFET is $3 \mathrm{pF}$, and the gate operating current, $I_{\mathrm{G}}$, is typically $1 \mathrm{pA}$. The equivalent input noise voltage, $e_{\mathrm{n}}$, is about $20 \mathrm{nV} \mathrm{Hz}^{-1 / 2}$ at $10 \mathrm{~Hz}, 10 \mathrm{nV} \mathrm{Hz}^{-1 / 2}$ at $100 \mathrm{~Hz}$ and less than $5 \mathrm{nV} \mathrm{Hz}^{-1 / 2}$ beyond $1 \mathrm{kHz}$, as given by the manufacturer's specifications. The differential gate-source voltage is $15 \mathrm{mV}$ at maximum and the offset voltage observed in the experiment is about $10 \mathrm{mV}$ due to this mismatch.

The output of the photogate is connected to the gate pin of Q2 within a distance of $5 \mathrm{~mm}$. Thus, the distributed coaxial-cable capacitance (at $70-100 \mathrm{pF} \mathrm{m}^{-1}$ ) typically encountered in the PC sampling technique is avoided. The total gate capacitance for the source follower is about $7 \mathrm{pF}$ due to both the photogate and $C_{\text {iss. }}$. Since the gate bias current of Q2 is negligible, the transferred PC charge is stored on the gate of this JFET. The only way for the stored charge to dissipate is through the dark resistance of the photogate itself. Since the voltage is equal to the charge divided by the input capacitance, the voltage level will build up more quickly by reducing the parasitic capacitance. If the on-period of modulation is long enough, an equilibrium voltage will be induced when the charge injection rate to this buffer capacitance balances the leakage current through the dark resistance. This buffer voltage will then be present at the output of the source follower. From the standpoint of the driving current, the source follower has a drain-source on-resistance of $140 \Omega$ and can supply a larger current to drive secondary amplifiers like a lock-in amplifier or digital averaging oscilloscope. The use of this source follower is equivalent to the optically strobed sampling head circuit pioneered by Lawton and Andrews [2]. However, the use of the matched JFET pair eliminates the need for a complex balanced circuit in the sampling head preamplifier.

\section{Results and discussion}

\subsection{Modulation bandwidth of photogates}

Figure 2 shows several temporally-resolved output signals from the photogate-source follower combination using gating laser powers of 2.8 and $1.4 \mathrm{~mW}$, and 100 and $10 \mu \mathrm{W}$. The signal under test was generated by the PX-D14 detector using $1 \mathrm{~mW}$ average optical power ( $25 \mathrm{pJ}$ per pulse) at a modulation frequency of $320 \mathrm{~Hz}$. The figure demonstrates that the temporal response of the photogate was independent of gating laser power over a wide range of gating power.

The modulation bandwidth of the sampling circuit with the source follower is investigated by observing the measured signal as the modulation frequency is adjusted from $1 \mathrm{~Hz}$ to $80 \mathrm{kHz}$. The temporal responses of these sampled signals in this range all have the same $10.1 \mathrm{ps}$ FWHM. The peak amplitudes of these waveforms are shown versus modulation frequency in Fig. 3 for three values of gating laser power. For comparison, the modulation bandwidth of the photogate without the source follower has also been studied and is shown in Fig. 3 using $1.4 \mathrm{~mW}$ gating laser power. At low modulation frequency, the amplitude of the sampled voltage approaches an equilibrium voltage where the injected charge into the input capacitance of the source follower balances the leakage current through the dark resistance of the photogate during each on-period of the modulation. The equilibrium voltage, $V_{\text {output }}$, at a $1 \mathrm{~Hz}$ modulation frequency increases by less than two times, from 280 to $420 \mathrm{mV}$, when the gating laser power increases 140 times from $10 \mu \mathrm{W}$ to $1.4 \mathrm{~mW}$. This saturation implies that the voltage at the input 


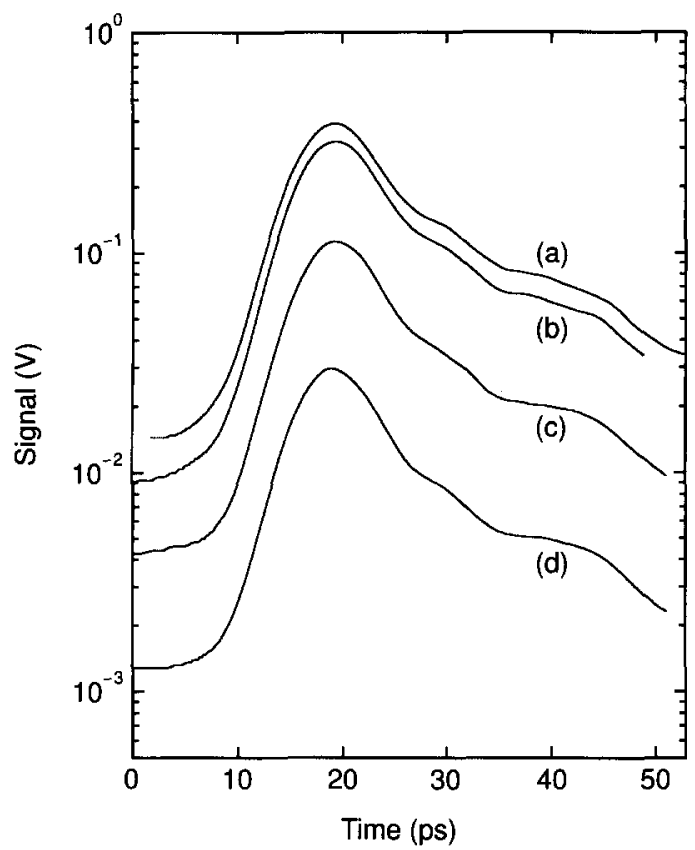

Figure 2 Temporally-resolved output signals from the photogate using gating laser powers of (a) $2.8 \mathrm{~mW}$, (b) $1.4 \mathrm{~mW}$, (c) $100 \mu \mathrm{W}$, and (d) $10 \mu \mathrm{W}$. The signal under test is generated using a pulsed laser with $1 \mathrm{~mW}$ average optical power ( $25 \mathrm{pJ}$ per pulse) modulated at $320 \mathrm{~Hz}$.

capacitance of the source follower indeed has the same order of magnitude as the signal, $V_{\text {signal }}$, from the PX-D14 detector for laser gating power above $10 \mu \mathrm{W}$.

The exact relation between $V_{\text {output }}$ and $V_{\text {signal }}$ can only be resolved numerically with the full knowledge of the detector and photogate impulse responses, since they are comparable in speed

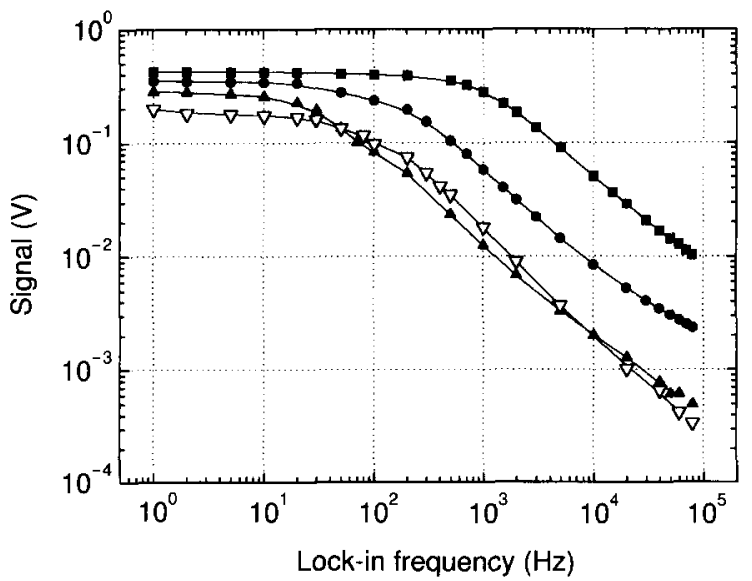

Figure 3 Modulation bandwidth of the photogate with the source follower using gating laser powers of $1.4 \mathrm{~mW}$ $(\mathbb{\square}), 100 \mu \mathrm{W}(\mathbf{O})$, and $10 \mu \mathrm{W}(\mathbf{A})$. The solid lines are a guideline to the eye. The modulation bandwidth using $1.4 \mathrm{~mW}$ gating laser power without the source follower is also shown $(\nabla)$. 
[18]. An analytic relation can only be analysed under the slow variation condition where the speed of the detector is much slower than that of the photogate. Under this condition, the bias voltage of the photogate during its 4 ps on-state period at a fixed excitation-/gate-pulse time delay can be approximated as a constant voltage. A quasi-d.c. approximation for the equilibrium voltage can then be determined from the photogate circuit diagram and given as:

$$
V_{\text {output }}=\frac{\eta P}{R^{-1}+\eta P} V_{\text {signal }}
$$

where $\eta$ is the photogate responsivity per voltage applied $\left(1.6 \times 10^{-3} \mathrm{~A} \mathrm{~W}^{-1} \mathrm{~V}^{-1}\right), P$ is the gating laser power, and $R$ is the effective load resistance of the sampling circuit. Although the slow variation condition does not hold strictly in this experiment, this quasi-d.c. approximation gives a consistent explanation of the experimental results. When the source follower is used, $R$ is the $150 \mathrm{M} \Omega$ dark resistance of the photogate, since the leakage current through the JFET is negligible. With the $1.4 \mathrm{~mW}$ gating laser power, $\eta P$ is much larger than $R^{-1}$, and $V_{\text {output }}$ is virtually equal to $V_{\text {signal }}$. This allows an absolute voltage measurement to be achieved at low modulation frequency. Without the source follower, $R$ is the $10 \mathrm{M} \Omega$ input impedance of the lock-in amplifier. Due to the current drain of this instrument, the equilibrium voltage without the source follower is smaller than that with the source follower when using the same gating power.

As the modulation frequency increases, the signal amplitude shown in Fig. 3 rolls off due to the finite modulation bandwidth of the sampling circuit. The parasitic capacitance of the photogate without the source follower is estimated to be about $200 \mathrm{pF}$, including the capacitance of a $1 \mathrm{~m}$ coaxial cable and the input capacitance of the lock-in amplifier. With the source follower, the dominant parasitic capacitance is reduced to the photogate capacitance and the commonsource input capacitance of the JFET, about $7 \mathrm{pF}$ total in this case. Without the source follower, the $3 \mathrm{~dB}$ frequency of the circuit response is about $100 \mathrm{~Hz}$ for a gating laser power of $1.4 \mathrm{~mW}$. After reducing the external parasitic capacitance by including the source follower, the $3 \mathrm{~dB}$ rolloffs are at 40 and $250 \mathrm{~Hz}$, and $1.5 \mathrm{kHz}$ for gating laser powers of 10 and $100 \mu \mathrm{W}$, and $1.4 \mathrm{~mW}$, respectively. Thus, by reducing the external parasitic capacitance, one has improved the modulation bandwidth of the photogate by about 15 times using the same $1.4 \mathrm{~mW}$ gating laser power. Beyond the $3 \mathrm{~dB}$ frequency, the modulated signal amplitude decreases proportionally with the modulation frequency since there is not enough time for the input capacitance of the source follower to reach its equilibrium voltage. The effective average resistance, $R_{\text {eff }}$, for the sampling circuit is given as:

$$
R_{\mathrm{eff}}^{-1}=R^{-1}+\eta P
$$

and the theoretical $3 \mathrm{~dB}$ frequency, $f_{3 \mathrm{~dB}}$, is given as:

$$
f_{3 \mathrm{~dB}}=\frac{R^{-1}+\eta P}{2 \pi C}
$$

or $360 \mathrm{~Hz}, 2.6$ and $36 \mathrm{kHz}$ for the photogate with the source follower using gating laser powers of 10 and $100 \mu \mathrm{W}$, and $1.4 \mathrm{~mW}$, respectively.

One should note that the experimental $3 \mathrm{~dB}$ frequencies in Fig. 3 are 9-24 times lower than the theoretical values. At the same time, the amplitude of the output signal is not linearly proportional to the gating laser power at modulation frequencies higher than $f_{3 \mathrm{~dB}}$. It increases only 25 times while the gating laser power increases 140 times from $10 \mu \mathrm{W}$ to $1.4 \mathrm{~mW}$. Since the voltage on the gate input capacitance is much smaller than the signal voltage from the detector, 
this indicates a saturation of the gating efficiency from a source other than the equilibrium voltage saturation as given by Equation 1. One reason for this is the high output impedance of the detector, which can only supply a limited current. The transient PC current, $I_{\mathrm{g}}$, from the photogate into the source follower in each sampling pulse can be given from the circuit diagram as:

$$
I_{\mathrm{g}}=\frac{Z_{\mathrm{S}}}{Z_{\mathrm{S}}+Z_{\mathrm{g}}} \frac{\left(V_{\text {signal }}-V_{\text {output }}^{\prime}\right)}{Z_{\mathrm{S}}}
$$

where $Z_{\mathrm{S}}$ is the output impedance of the detector, $Z_{\mathrm{g}}$ is the on-state impedance of the photogate, $V_{\text {signal }}$ is the part of the signal from the detector sampled by the $4 \mathrm{ps}$ on-state period of the photogate at a fixed excitation-/gate-pulse delay time, and $V_{\text {output }}^{\prime}$ is the transient voltage at the gate pin of the JFET Q2 during the on-period of the modulation. At high modulation frequency, such that the amplitude of $V_{\text {output }}^{\prime}$ is always much less than $V_{\text {signal }}, I_{\mathrm{g}}$ can be approximated as:

$$
I_{\mathrm{g}}=\frac{V_{\text {signal }}}{Z_{\mathrm{S}}+Z_{\mathrm{g}}}
$$

where the maximum current is limited by the output impedance of the detector, which is $100 \Omega$ in this experiment. This circuit limitation of the maximum photogate current could explain the relatively low experimental $3 \mathrm{~dB}$ frequencies at high gating laser power. Other possible limitations, due to the screening effect of the high-density PC space charge or the finite contact resistance between the metal-LT-GaAs interface [19], could further reduce the maximum photogate current. In general, Fig. 3 shows that the gating efficiency of the photogate is not linearly proportional to the gating laser power above $10 \mu \mathrm{W}$ over all modulation frequencies from $1 \mathrm{~Hz}$ to $80 \mathrm{kHz}$. Thus, the signal-to-noise ratio might not be increased by simply increasing the gating laser power. This issue will be discussed with experimental results in the following sections.

Finally, the conventional way to calibrate the sensitivity of the photogate is to apply a modulated electrical signal of known amplitude to the photogate and measure the photogate output on the lock-in amplifier [9]. The dependence of the measured signal on the output impedance of the device-under-test and the modulation frequency has demonstrated that this calibration method must be employed carefully, and that absolute voltage measurements are only possible at low modulation frequencies.

\subsection{Noise limit}

In general, the noise in a PC sampling measurement may arise from both the signal source and the photogate. By reducing the output signal from the PX-D14 detector in the experiment, the noise limit of the photogate can be studied without influence from the detector noise. Figure 4 shows the temporal response of signals generated using $50 \mathrm{nW}$ of optical pumping power (1.2 fJ per pulse), modulated at frequencies of 50 and $500 \mathrm{~Hz}$, and 5 and $50 \mathrm{kHz}$, and measured using $1.4 \mathrm{~mW}$ of gating laser power and the source follower. The dwell time for each sampling step at a fixed excitation-/gate-pulse time delay and the integration time constant are both $1 \mathrm{~s}$. The temporal response of the sampled signal at $50 \mathrm{kHz}$ modulation frequency is $10.2 \mathrm{ps}$ FWHM, nearly the same as those shown in Fig. 2. The amplitude of the signal voltage is about $30 \mu \mathrm{V}$ for a modulation frequency of $50 \mathrm{~Hz}$ (Fig. 4a). This voltage should be essentially the same as the actual output signal from the PX-D14 detector, via the explanation for Fig. 3 at low modulation frequencies. The root-mean-square noise, $V_{\mathrm{Nrms}}$, is calculated from a trace of the signal output measured with a $1 \mathrm{~s}$ integration time during a $1 \mathrm{~min}$ period in which the gating 
laser pulses to the photogate are synchronized to arrive $30 \mathrm{ps}$ ahead of the excitation pulses to the detector, i.e. similar to a time at $0 \mathrm{ps}$ on the baselines of the traces in Fig. 4 . At $50 \mathrm{kHz}$ modulation frequency, the maximum signal level on the lock-in amplifier is reduced to $1 \mu \mathrm{V}$ due to the finite modulation bandwidth of the sampling circuit. However, the noise is reduced even more. Thus, the signal-to-noise ratio is increased from 13 to 270 when the modulation frequency is increased from $50 \mathrm{~Hz}$ to $50 \mathrm{kHz}$.

Figure 5 shows the peak amplitude of the output signal and $V_{\mathrm{Nrms}}$ versus modulation frequency for the $30 \mu \mathrm{V}$ output from the PX-D14 using gating laser powers of 10 and $100 \mu \mathrm{W}$ and $1.4 \mathrm{~mW}$ and the source follower. The temporal responses of these sampled signals are all approximately $10.1 \mathrm{ps}$ FWHM, although for modulation frequencies below $500 \mathrm{~Hz}$ the pulses become broader due to their poor signal-to-noise ratios. As expected, when the photogate responses at each of these gating laser powers are compared with those of Fig. 3, the same modulation bandwidths are observed regardless of the signal amplitude changing over four orders of magnitude. Furthermore, the ratios of the signals using these three gating powers over the span of modulation frequencies are the same for Figs 3 and 5. This implies the modulation bandwidth of the photogate circuit is mainly determined by the effective RC time constant of the sampling circuitry, as defined by Equation 3. However, one does notice the signal increases only $1.3 \times 10^{4}$ times, from $30 \mu \mathrm{V}$ to $390 \mathrm{mV}$, when the laser excitation power to the PX-D14 increases $2.0 \times 10^{4}$ times from $50 \mathrm{nW}$ to $1 \mathrm{~mW}$. It is believed that this subtle nonlinearity is due to the photogate efficiency instead of the detector efficiency, as the photogate is biased by the small electrical signal between $30 \mu \mathrm{V}$ and $390 \mathrm{mV}$ and the detector is always biased by a $5 \mathrm{~V}$, low-impedance source. That is, the photoexcited charges inside the photogate biased at $30 \mu \mathrm{V}$ move much slower and are more likely to be influenced by the metal-LT-GaAs contact properties than those biased at $390 \mathrm{mV}$.
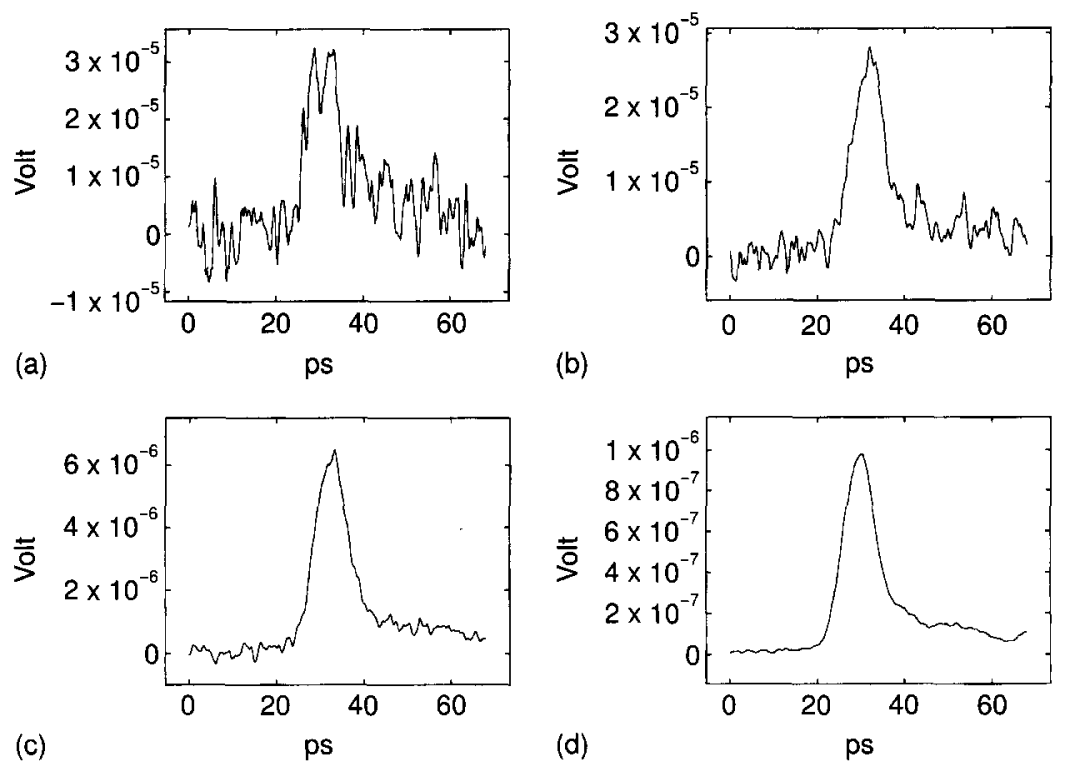

Figure 4 Temporal response of signal generated using $50 \mathrm{nW}$ average optical pump-power with modulation frequencies of (a) $50 \mathrm{~Hz}$, (b) $500 \mathrm{~Hz}$, (c) $5 \mathrm{kHz}$, and (d) $50 \mathrm{kHz}$. The source follower and $1.4 \mathrm{~mW}$ of gating laser average power are utilized. 
At low modulation frequency, the photogate noise is roughly proportional to the square root of the gating laser power. For example, the noise increases ten times at a modulation frequency of $200 \mathrm{~Hz}$ and eight-and-a-half times at $500 \mathrm{~Hz}$ for a factor of 140 increase of the gating laser power from $10 \mu \mathrm{W}$ to $1.4 \mathrm{~mW}$. This noise also has a $1 / f$ frequency dependence and approaches a minimum around $4 \mathrm{nV} \mathrm{Hz}^{-1 / 2}$, the noise limit of the JFET at high modulation frequency. In comparison with this data, Fig. 6 shows the measured voltage signal and noise of the photogate without the source follower using $1.4 \mathrm{~mW}$ of gating laser power. The response shows that this device has the same signal amplitude as that of the photogate with the source follower using only $10 \mu \mathrm{W}$ of gating laser power. On the other hand, the amplitude of the noise spectrum is the same as that in Fig. 5 for $1.4 \mathrm{~mW}$ of gating laser power.

\subsection{Signal-to-noise ratio}

Figure 7 shows the signal-to-noise ratios for the measurements in Figs 5 and 6. The signal-tonoise ratio without the source follower is limited by the small modulation bandwidth due to a large external parasitic capacitance. It has little variation over the range of modulation frequency since the signal and noise both have a $1 / f$ frequency dependence. With the source follower, the bandwidth of the photogate circuit allows the use of higher modulation frequencies and a better signal-to-noise ratio is achieved. For the same $1.4 \mathrm{~mW}$ of gating laser power, the signal-to-noise ratio is increased by over 50 times at a $50 \mathrm{kHz}$ modulation frequency. To increase further the signal-to-noise ratio, gating laser powers greater than $1.4 \mathrm{~mW}$ have also been used.

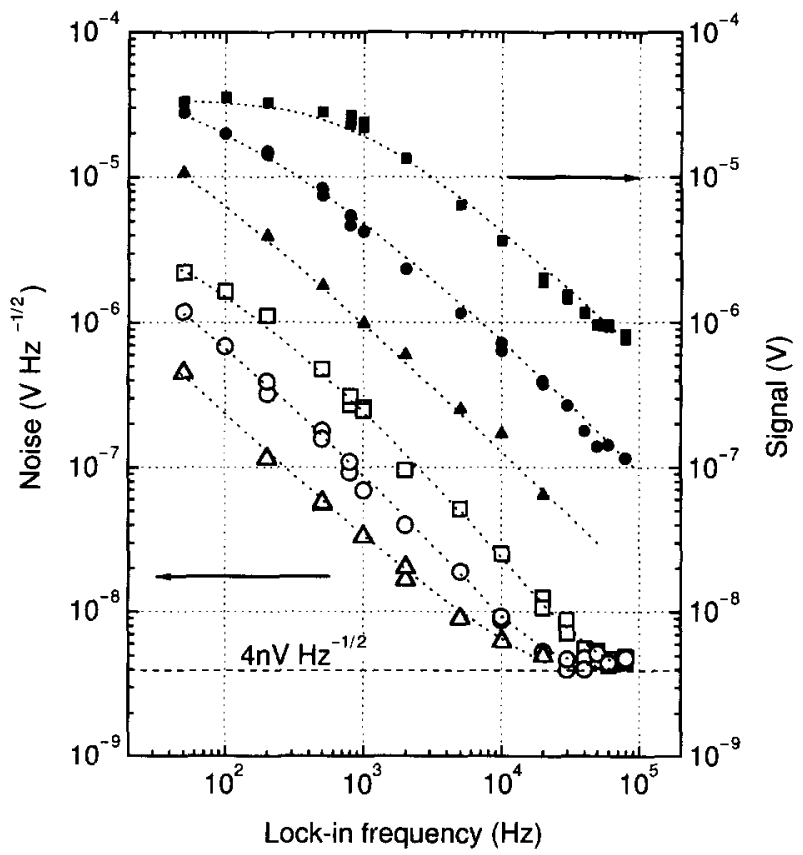

Figure 5 Measured voltage signal and noise for a modulated $30 \mu \mathrm{V}$ signal generated using $50 \mathrm{nW}$ average optical pump-power and the source follower. The solid symbols are the peak amplitudes of the signais and the open symbols are the noise for different gating laser powers and modulation frequencies: $(\mathbb{G}) 1.4 \mathrm{~mW}$, (e) $100 \mu \mathrm{W}$, and (4) $10 \mu \mathrm{W}$. The dashed lines are a guide to the eye. 


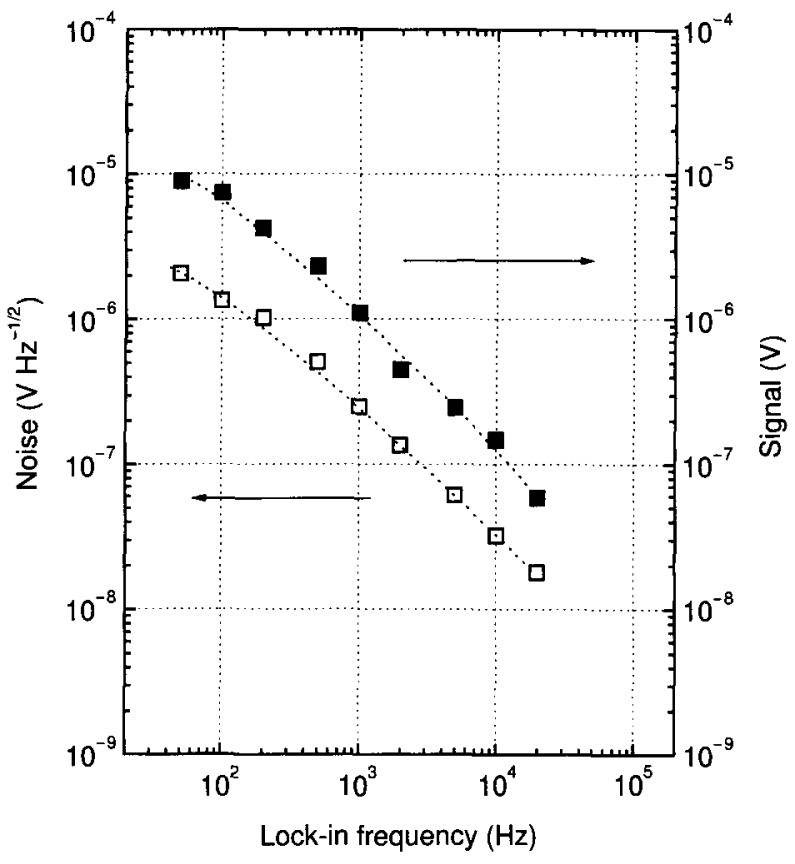

Figure 6 Measured voltage signal $(\square)$ and noise $(\square)$ for a modulated $30 \mu \mathrm{V}$ signal using $1.4 \mathrm{~mW}$ gating laser power without the source follower. The dashed lines are a guide to the eye.

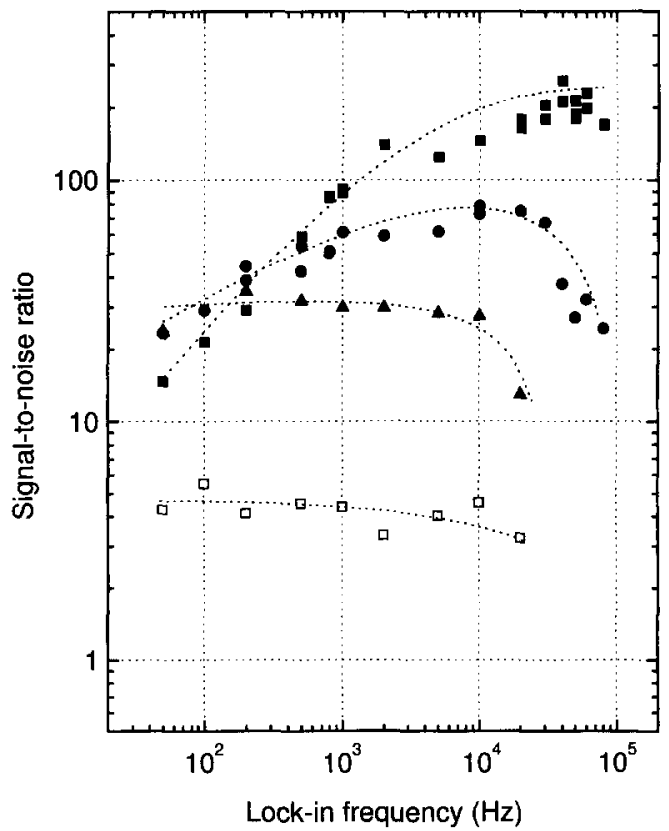

Figure 7 Signal-to-noise ratios for the measurements of Figs 5 and 6 . The symbols are the same as in Figs 5 and 6. 
However, the output signal amplitude does not increase greatly as the gating laser power increases. For example, at a modulation frequency of $50 \mathrm{kHz}$, the sampling signal increased only 1.7 times as the gating laser power was raised from 1.4 to $6 \mathrm{~mW}$. There is no gain in signal-to-noise ratio since the noise also increases by a factor of two. Higher gating laser powers were not tested due to the risk of damaging the photogate.

It is known that the noise of the photogate does not arise from the dark current, because there is no dark current when the external bias is removed. Also, the observed noise is not thermal (white noise), but rather it has a $1 / f$ frequency dependence. In addition, the authors have already shown in Section 3.2 that the photogate noise increases with the square root of the gating laser power. Thus, one is led to the conclusion that the photogate noise is dominated by the $1 / f$ noise of the gating laser amplitude fluctuations. One possible noise mechanism arises from the photovoltaic effect due to the illumination of the metal-LT-GaAs contact by the gating laser pulses. To confirm and reduce this photovoltaic noise effect, it is necessary to verify the nature of the metal contact on the LT-GaAs and to make it an ohmic contact. However, this has proved to be difficult due to the compensating effect of the high density of mid-gap defect states in LT-GaAs. However, one already has a photogate noise which is close to the noise limit of the lock-in amplifier. The practical way to increase the signal-to-noise ratio is to reduce further the parasitic capacitance, thus increasing the modulation bandwidth without increasing the gating laser power.

It is worthwhile to note here the NEP of the photogate in this PC sampling experiment. For instance, Fig. 8 shows the temporal response after a $5 \mathrm{nW}$ excitation of PX-D14 detector at a $50 \mathrm{kHz}$ modulation frequency measured with the source follower and $1.4 \mathrm{~mW}$ gating laser power. The noise measured is $4.5 \mathrm{nV} \mathrm{Hz}^{-1 / 2}$, and the signal-to-noise ratio is still 25 . This implies a $200 \mathrm{pW}$ NEP. This result can be compared with that of Chen et al. [6]. In order to achieve a $500 \mathrm{pW}$ NEP, it was necessary to use a much finer, $0.2 \mu \mathrm{m}$ finger width and spacing for the MSM structure and to achieve a responsivity of $0.1 \mathrm{~A} \mathrm{~W}^{-1}$ for both the detector and photogate. This highlights the improvement in the performance and cost of the PC sampling

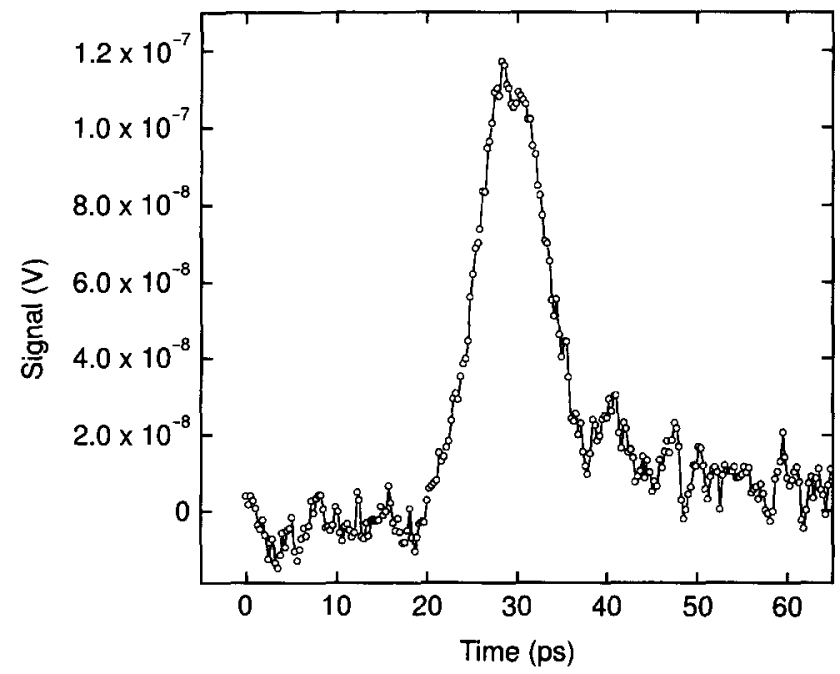

Figure 8 Temporal response of a $5 \mathrm{nW}$ signal from the PX-D14 detector measured with the source follower at a modulation frequency of $50 \mathrm{kHz}$ and $1.4 \mathrm{~mW}$ gating laser power. 
technique by reducing the external parasitic capacitance and increasing the modulation bandwidth.

\subsection{Invasiveness of photogates}

Using a high laser power to illuminate a photogate with a large parasitic capacitance (i.e. with no source follower) can have a disadvantage in terms of invasiveness. For example, in the experiments using $1.4 \mathrm{~mW}$ of gating laser power, the average resistance of the photogate was reduced to $430 \mathrm{k} \Omega$. The on-state resistance was then estimated to be about $140 \Omega$ from the duty cycle of 3050 ( $4 \mathrm{ps}$ on-state to $12.1 \mathrm{~ns}$ off-state). A photogate with an impedance this low would be too invasive if it were implemented on a probe for measurements in many digital circuits. In this case, $80 \mathrm{fC}$ of charge will be drained away from a $3 \mathrm{~V}$ state during a period of $4 \mathrm{ps}$. This invasiveness is also due to the external parasitic capacitance of the sampling circuit. In addition, a photogate with an on-state resistance lower than the impedance of a device-under-test will have inferior signal-to-noise ratio as discussed above. By reducing the external parasitic capacitance, the same signal level can be achieved with less invasiveness using lower gating laser power. For example, from Figs 5 and 6, the same signal level is obtained using only $10 \mu \mathrm{W}$ of gating laser power as is found using $1.4 \mathrm{~mW}$ of gating laser power without the source follower. The on-state resistance is increased 140 times from $140 \Omega$ to $20.5 \mathrm{k} \Omega$, while the photogate noise is reduced about seven times. Furthermore, a low gating-power photogate will help to reduce the power requirements of the ultrafast laser. Long-term stability of the photogate is also an advantage of the photogate using low gating laser power since less optical energy will be deposited onto the photogate.

\section{Conclusions}

In conclusion, the modulation bandwidth and noise limits of a photogate have been studied by reducing its external parasitic capacitance with a novel JFET source follower. Photogate noise is measured to be as low as $4 \mathrm{nV} \mathrm{Hz}^{-1 / 2}$. The dominant photogate noise source is determined to be the gating laser power noise due to the photovoltaic current. There is little gain in signal-tonoise ratio after the impedance of the photogate becomes lower than that of the device-undertest. The modulation bandwidth of the PC sampling circuit is limited by the parasitic capacitance. The best way to improve the signal-to-noise ratio is to reduce the parasitic capacitance of the photogate after the photogate efficiency saturates at high gating laser power. With the integration of the source follower, the external parasitic capacitance of the photogate is reduced and the modulation bandwidth is increased 15 times. The leakage of the charge associated with the signal is also reduced by the extremely high input impedance of the JFET source follower. The signal-to-noise ratio is increased in total by a factor of more than 45 . High performance in a photogate in regard to invasiveness and gating efficiency has been achieved simultaneously while maintaining a picosecond temporal response and enhancing the signal-to-noise performance. Laser powers as low as $10 \mu \mathrm{W}$ have been demonstrated to be sufficient for driving the photogate. The integration of the photogate with the source follower will help speed the development of compact, economical PC sampling probes and measurement systems with enhanced performance.

\section{Acknowledgements}

This research was sponsored by the Air Force Office of Scientific Research, Air Force Material Command, USAF, under grant number DOD-G-F49 620-95-1-0227. The US Government is authorized to reproduce and distribute reprints for governmental purposes notwithstanding 
any copyright notation thereon. Support was also provided by the National Science Foundation through the Center for Ultrafast Optical Science under STC PHY 8920 108. The views and conclusions contained herein are those of the authors and should not be interpreted as necessarily representing the official policies or endorsements, either expressed or implied, of the Air Force Office of Scientific Research or the US Government.

\section{References}

1. J. A. VALDMANIS and G. MOUROU, IEEE J. Quantum Electron. 22 (1986) 69.

2. R. A. LAWTON and J. R. ANDREWS, IEEE Trans. on Instrum. \& Meas. 25 (1976) 56.

3. D. H. AUSTON, A. M. JOHNSON, P. R. SMITH and J. C. BEAN, Appl. Phys. Lett. 37 (1980) 371.

4. Y. CHEN, S. WILLIAMSON and T. BROCK, Appl. Phys. Lett. 59 (1991) 1984.

5. F. W. SMITH, H. Q. LE, V. DIADIUK, M. A. HOLLIS, A. R. CALAWA, S. GUPTA, M. FRANKEL, D. R. DYKAAR, G. A. MOUROU and T. Y. HSIANG, Appl. Phys. Lett. 54 (1989) 890.

6. Y. CHEN, S. WILLIAMSON and T. BROCK, Appl. Phys. Lett. 64 (1994) 551.

7. J. KIM, S. WILliaMSON, J. NEES, S. WAKANA and J. WHITAKER, Appl. Phys. Lett. 62 (1993) 2268.

8. T. PREIFER, H.-M. HEILIGER, E. STEIN VON KAMIENSKI, H. G. ROSKOS and H. KURZ, J. Opt. Soc. Am. B 11 (1994) 2547.

9. J. KIM, J. SON, S. WAKANA, J. NEES, S. WILliAMSON, J. WHITAKER and G. MOUROU, in OSA Proceedings on Ultrafast Electronics and Optoelectronics, edited by J. Shah and U. Mishra, vol. 14 (Optical Society of America, Washington, DC, 1993) p. 224.

10. J. NEES, D. CRAIG, S. HAMA and S. WAKANA, in Ultrafast Electronics and Optoelectronics, vol. 13, OSA Technical Digest Series (Optical Society of America, Washington, DC, 1995) p. 172.

11. S. WEISS, D. F. OGLETREE, D. BOTKIN, M. SALMERON and D. S. CHEMLA, Appl. Phys. Lett. 63 (1993) 2567.

12. D. H. AUSTON, IEEE J. Quantum Electron. 19 (1983) 639.

13. D. H. AUSTON, in Ultrashort Laser Pulses: Generation and Applications, edited by W. Kaiser, 2nd edn (SpringVerlag, New York, 1993) p. 183.

14. K. J. WEINGARTEN, M. J. W. RODWELL and D. M. BLOOM, IEEE J. Quantum Electron. 24 (1988) 198.

15. S. L. HUANG, E. A. CHAUCHARD, C. H. LEE, H. L. HUNG, T. T. LEE and T. JOSEPH, IEEE Trans. Microwave Theory Technol. 40 (1992) 2312.

16. J. R. HWANG, H. J. CHENG, J. F. WHITAKER and J. V. RUDD, Appl. Phys. Lett. 68 (1996) 1464.

17. E OXNER, Designing with Field-Effect Transistors, 2nd edn (McGraw-Hill, New York, 1990).

18. D. R. GRISCHKOWSKY, M. B. KETCHEN, C. C. CHI, I. N. DULING, III, N. J. HALAS, J. M. HALBOUT and P. G. MAY, IEEE J. Quantum Electron. 24 (1988) 221.

19. M. Y. FRANKEL, J. F. WHITAKER, G. A. MOUROU, F. W. SMITH and A. R. CALAWA, IEEE Trans. Electron Devices 37 (1990) 2493. 